\title{
Abstracts of Theses Approved for the MSc and PhD Degrees at the Faculty of Medicine, Health Sciences Centre, Kuwait University, Kuwait
}

1

\section{The Role of Chlamydia trachomatis, Genital Mycoplasmas, and Genital Ureaplasmas in Male Infertility in Kuwait}

Amani H.E. Al-Fadli

Department of Microbiology, Faculty of Medicine, Kuwait University, Kuwait

The prevalence of infertility appears to be on the increase globally. This may, in part, be a result of better diagnosis of infertility. Male infertility accounts for $30 \%$ of infertility in couples and is a contributing factor in another $20-30 \%$ of couples. Male genital tract infections and inflammation have been associated with $8-35 \%$ of male infertility cases in various studies. Sexually transmitted infections are caused by heterogeneous microorganisms that infect men and may be transmitted to their sexual partners if not treated. The potential mechanisms of these pathogens on fertility may be by a direct negative effect that they have on spermatozoa or an indirect effect via infection leading to inflammatory obstruction of the tubules and/or epithelial damage resulting in impaired spermatogenesis. The main aim of this study was to investigate the potential role that some selected uropathogens, like Chlamydia trachomatis, genital mycoplasmas, and genital ureaplasmas, may play in male infertility in Kuwait.

A total of 315 semen samples were collected from 2 major government institutions: the Maternity Hospital and the Gynecology Department of the Faculty of Medicine in Kuwait. They were subjected to semen analysis as part of the workup for infertility investigations. Semen analysis was performed on semen samples according to the WHO manual (1992). Semen analysis parameters included seminal volume, $\mathrm{pH}$, sperm concentration $\left(\times 10^{6} \%\right.$ $\mathrm{ml}$ ), vitality, percentage motile, motility gradation, WBC, and normal and abnormal sperm morphology. The presence of $M y$ coplasma hominis and Ureaplasma urealyticum was investigated using the MYCOFAST Evolution 3 test for culture. The presence of uropathogens was also investigated using specific PCR primers for detecting the genes for M. hominis, U. urealyticum, Mycoplasma genitalium, and C. trachomatis on all specimens. We found a highly significant difference between the infertile and control groups with respect to sperm motility, count, morphology, and vitality. The rates of oligozoospermia were 13 and $0 \%$ in the infertile and control groups, respectively $(\mathrm{p}<0.001)$, while the azoospermia rates were $0 \%$ in the control group and $13 \%$ in the infertile group $(\mathrm{p}<0.001)$. All of the semen samples in the control group were normozospermic (100\%). The relationship between different age groups and semen parameters showed a highly significant difference $(\mathrm{p}<0.05)$ between the infertile and control groups in the age groups 30-34, 35-39, and $>55$ years with respect to sperm motility. Our study demonstrated an overall high prevalence of $M$. hominis, U. urealyticum, and C. trachomatis and a low prevalence of M. genitalium in both groups, with no significant difference. However, analysis of sperm parameters in the infertile group in relation to the presence of uropathogens showed a significant association between abnormal sperm parameters in those with sperms positive for M. hominis, C. trachomatis, and $U$. urealyticum. For instance, sperm vitality, count, and motility were significantly affected $(\mathrm{p}<0.001)$ in the presence of M. hominis, C. trachomatis, and U. urealyticum in comparison to negative semen samples. In conclusion, uropathogens appear to have a potential effect on sperm quality and parameters which are usually identified as potential drivers of male infertility. Further studies are needed to examine other uropathogens and the exact effect of these pathogens on sperm parameters and morphology.

Support: Supported by a fellowship from the College of Graduate Studies and Kuwait University Research Administration grant No. YM 03/09.

Noura Al-Sweih (Supervisor)

V.O. Rotimi (Co-Supervisor)

\section{KARGER}

Fax +4161306 1234

E-Mail karger@karger.ch

www.karger.com
C 2011 S. Karger AG, Basel

$1011-7571 / 11 / 0206-0590 \$ 38.00 / 0$

Accessible online at:

www.karger.com/mpp 


\section{2}

A Comparative Evaluation of Mitogillin, Galactomannan, (1 $\rightarrow 3)-\beta-D-g l u c a n$, and Aspergillus fumigatus DNA in the Diagnosis of Experimental Invasive Aspergillosis

\section{Ali Abdulreda Mohammed Al-Shaikh}

Department of Microbiology, Faculty of Medicine, Kuwait University, Kuwait

The common environmental mold Aspergillus fumigatus is the main causative agent of invasive pulmonary aspergillosis (IPA) in neutropenic/severely immunocompromised patients and is associated with very high mortality rates. Early and accurate diagnosis of IPA is crucial for a better prognosis and for the selection of appropriate antifungal therapy. An experimental inhalation model of IPA was developed in rats, and the efficacy of 4 noninvasive markers $[(1 \rightarrow 3)-\beta$-D-glucan (BDG), galactomannan (GM), $A$. fumigatus DNA, and 18-kDa antigen (mitogillin; MIT)] was evaluated in serum and bronchoalveolar lavage (BAL) specimens for the early diagnosis of IPA. BDG and GM were detected using Fungitel and Platelia Aspergillus EIA kits, respectively, according to the instructions supplied by the kit manufacturers. A. fumigatus DNA was detected by developing a sensitive, single-step PCR amplification protocol. To detect MIT protein in serum, BAL, or urine by immunoblotting, polyclonal anti-MIT antibodies were generated first by cloning and expression of the MIT gene of $A$. fumigatus in Escherichia coli and purification of the recombinant MIT protein to homogeneity. IPA was successfully developed in rats immunosuppressed with steroid. Until 5 days postinfection, all of the animals were uniformly positive for the lung tissue culture and $\mathrm{KOH}$-calcofluor microscopy. Although MIT protein was not detected in serum, BAL, or urine specimens from any of the experimentally infected rats, 14 of $30(47 \%)$ and 27 of $30(90 \%)$ serum and BAL specimens, respectively, were positive for the remaining 3 markers (BDG, GM, and A. fumigatus DNA). The specificity of the tests was $100 \%$ as none of the sera or BAL specimens of the 12 control rats yielded positive results for BDG, GM, or A. fumigatus DNA detection. Amplification of the rat $18 \mathrm{~S}$ rRNA gene showed that the lack of A. fumigatus DNA detection in negative samples was not due to the presence of PCR inhibitors. Expression and secretion of MIT protein was detected after $24 \mathrm{~h}$ of growth in Sabouraud dextrose or potato dextrose broth, and its amount increased progressively until $96 \mathrm{~h}$ in culture medium of in vitro grown A. fumigatus cells. The expression and secretion of MIT protein was both delayed and reduced with an increasing concentration of rat serum added to Sabouraud dextrose broth during in vitro growth of A. fumigatus. Our data show that BAL is a superior specimen compared to serum, and combined detection of BDG, GM, and A. fumigatus DNA provides a sensitive diagnosis of IPA in an experimental animal model. The downregulation of MIT protein expression and secretion by serum component(s) is consistent with our inability to detect MIT protein in any of the serum, BAL, or urine specimens of rats infected with A. fumigatus.

Support: Supported by a fellowship from the College of Graduate Studies and Kuwait University Research Administration grant No. YM 07/07.

Prof. Suhail Ahmad (Supervisor)

Prof. Ziauddin Khan (Co-Supervisor)
3

Immunogenicity and Pathogenesis of RD1 Region Proteins of Mycobacterium tuberculosis Expressed in Mycobacterium smegmatis and Mycobacterium bovis Bacillus Calmette-Guérin

Salwa Khalid Al-Mutairi

Department of Microbiology, Faculty of Medicine, Kuwait University, Kuwait

Introduction and Objective: Tuberculosis (TB) is a global infectious disease problem, with about one third of the world population being infected, 9 million people developing the active disease, and 1.7 million dying of TB each year. The existing vaccine against TB, which is an attenuated strain of Mycobacterium bovis Bacillus Calmette-Guérin (BCG), does not provide consistent protection against the disease. Thus, there is an urgent need to develop new vaccines against TB. Genetic analysis of BCG has shown the deletion of the region of difference (RD)1, which is present in pathogenic Mycobacterium tuberculosis and M. bovis and encodes $14 M$. tuberculosis-/M. bovis-specific proteins from an equal number of open reading frames (ORFs). Recently, it has been shown that 4 of these ORFs, i.e. ORF3 (rv3872), ORF5 (rv3873), ORF6 (CFP10), and ORF7 (ESAT6), encode proteins that are major targets for recognition by protective Thelper (Th)1 cells in infected humans and animals. This study aimed to study the immunogenicity and pathogenesis of these ORFs after expression in two mycobacterial hosts, i.e. Mycobacterium smegmatis and M. bovis BCG. Methods: DNA corresponding to the above mentioned genes were cloned in 3 shuttle plasmid vectors (pSMT3, pDE22, and pAU151), and the recombinant plasmids were used to transform Escherichia coli. The recombinant plasmids containing the target genes were purified from the transformed E. coli cells using the miniprep method and electroporated into $M$. bovis BCG and $M$. smegmatis. The transformed mycobacterial cells were selected by growing on selective media under the pressure of an appropriate antibiotic, i.e. hygromycin. Polymerase chain reaction was used to detect the presence of desired inserts in the growing organisms, and the identity of amplified DNA was confirmed by size identification using agarose gel electrophoresis. The immunogenicity and pathogenesis of the transformed mycobacterial cells were tested in mice and guinea pig models of TB. Results: Only M. smegmatis could be transformed with the minipreps of recombinant plasmids. Furthermore, the studies to detect the expression of RD1 proteins in recombinant $M$. smegmatis suggested that only ORF3 protein $(10 \mathrm{kDa})$ was expressed in cells transformed with pDE22/ORF3, as detected by Western immunoblot analysis. Spleen cells from mice immunized with recombinant $M$. smegmatis, transformed with pDE22/ORF3, proliferated in response to the peptide pool of ORF3 (ORF3mix) and its individual peptides, except peptide 6 . In addition, guinea pigs immunized with recombinant $M$. smegmatis-pDE22/ORF3 showed delayed type hypersensitivity skin responses to the sonicates of $M$. smegmatis as well as to ORF3mix and individual peptides of ORF3. Furthermore, the experiments to determine the pathogenesis of recombinant mycobacteria showed that $M$. smegmatis-pDE22/ORF3 was cleared from the internal organs at least as fast as wild-type M. smegmatis. Conclusion: This study confirms in vitro and in vivo expres- 
sion as well as the immunogenicity of ORF3 in recombinant $M$. smegmatis, without increased pathogenesis, and suggests its potential for developing a safer vaccine against TB.

Prof. Abu Salim Mustafa (Supervisor)

Dr. Hanady A. Amoudy (Co-Supervisor)

\section{4 \\ Localization of $\mathrm{Na}-\mathrm{H}$ Exchanger Isoform-8 in Lipid Membrane Microdomains (Rafts) in the Apical Domain of Epithelial Cells and Its Role in Experimental Colitis}

Amna Abdulwahab Al-Shamali

Department of Biochemistry, Faculty of Medicine, Kuwait University, Kuwait

$\mathrm{Na}-\mathrm{H}$ exchanger (NHE) is a membrane protein which performs electroneutral uptake of $\mathrm{NaCl}$ and water from the gastrointestinal tract. Among various NHE isoforms which show tissue/cell selective expression, NHE-1 and NHE-3 are located on the basolateral and apical domains of the epithelial cells, respectively. While NHE-1 mainly regulates $\mathrm{pH}_{\mathrm{i}}$, the NHE-3 isoform is mostly implicated in the absorption of $\mathrm{NaCl}$. Gene knockout studies and cloning techniques have confirmed the presence of several NHE isoforms on the same locus in a cell. For example, the NHE- 8 isoform is also localized along with NHE-2 and NHE3 in the apical domain of renal and intestinal epithelial cells. Although NHE-8 performs the same function as the other apical isoforms such as NHE-3, the former has shown unique kinetic properties. For example, NHE- 8 has a lower affinity for $\mathrm{Na}^{+}$ion concentration as compared to NHE-3. Furthermore, the abundance of NHE-8 tends to decline following maturation through the action of hormonal and growth factors indicating a developmental regulation. Unlike NHE-3, NHE-8 does not respond to cAMP and is not coupled to G-protein regulation. Many studies have shown involvement of NHE isoforms in a number of diseases including inflammatory bowel disease. In this study we investigated the mechanism of NHE-8 expression regulation in experimental colitis induced in adult rats by intracolonic instillation of 2,4,6-trinitrobenzenesulfonic acid (TNBS). Our previous studies showed maximum inflammatory responses on day 5 post-TNBS. Therefore this condition was selected in this study. In order to investigate the role of inflammatory mediators, the effect of dexamethasone was also studied. Colonic segments were collected from 5-day post-TNBS treatment and were used to estimate NHE- 8 protein by ECL Western blot analysis and mRNA levels by a competitive RT-PCR method developed in this study. Characterization of colitis was performed by measuring myeloperoxidase (MPO) activity, malondialdehyde levels, and H\&E histological staining. To investigate the underlying mechanism, we studied the localization of NHE-8 in the lipid raft fractions prepared from crude microsomes of noncolitis rat colonic segments. Lipid raft fractions were prepared by separating the triton X-100-solubilized crude microsomes on a discontinuous-sucrose gradient. Ten fractions starting from the top of centrifuge tube were collected, and protein and cholesterol contents, NHE-8,
NHE-3, caveolin-1, flotillin-1, and actin protein levels were estimated. The NHE-8 protein level was significantly decreased in experimental colitis and was not reverted by dexamethasone treatment. Abundance of NHE- 8 mRNA and actin protein, a housekeeper, remained unchanged in the test conditions. However, dexamethasone significantly decreased the level of MPO activity, malondialdehyde, and infiltration of inflammatory cells in inflamed colon. With regard to localization, NHE-8 protein was not detected in the lipid raft fractions but was present only in the nonlipid raft fractions similar to actin. Interestingly, NHE3 was distributed in both the lipid raft and nonraft fractions. Raft membrane fractions were enriched in the markers flotillin-1 and caveolin-1 and cholesterol. Our findings suggest that downregulation of NHE-8 protein in inflamed adult rat colon is regulated posttranscriptionally and contributes to the pathogenesis of colitis. Unique localization of NHE-8 showing absence in the lipid raft fractions suggests that the NHE- 8 isoform is regulated differently in inflammation than the other apical isoforms such as NHE-3 and is independent of regulation through cAMP, G-protein, or endosome recycling.

Islam Khan (Supervisor)

\section{Role of $\mathrm{Na}^{+} / \mathrm{H}^{+}$Exchanger in Resveratrol-Induced Growth Inhibition of Human Breast Cancer Cells}

Heba Mehdawi

Department of Biochemistry, Faculty of Medicine, Kuwait University, Kuwait

Resveratrol (RSVL), a natural phytoalexin, is found in many plant species and fruits including red grape skin, and it possesses anticancer properties. However, the mechanism of its antitumor effects remains poorly understood. The present study was designed to investigate the mechanism of action of RSVL using human breast cancer cell lines MDA-MB-231 and MCF-7. We analyzed the effect of RSVL on cell proliferation and viability, cell morphology, and the cell cycle. Our results showed that RSVL caused suppression of cell growth and viability. Growth suppression was due to apoptosis as detected by the appearance of a sub G1 fraction by flow cytometry. RSVL treatment caused morphological changes associated with apoptosis including increased cell size and detachment in both cell lines. Furthermore, to understand the mechanism of the effect of RSVL, we examined the expression of $\mathrm{Na}^{+} / \mathrm{H}^{+}$exchanger (isoforms 1 and 3), which regulates intracellular $\mathrm{pH}$, cell volume, and shape. Using immunofluorescence assay and ECL Western blot analysis, we showed for the first time that treatment with RSVL resulted in an increased expression of NHE1 and NHE3 proteins. In other cellular systems, it is well known that overexpression of NHE1 and NHE3 tends to increase the intracellular concentration of $\mathrm{Na}^{+}$and $\mathrm{Ca}^{2+}$ and results in apoptosis or cell necrosis. In conclusion, RSVL seems to exert its growth inhibitory/apoptotic effect on MDA-MB-231 and MCF-7 cells by increasing the expression of NHE1 and NHE3 proteins.

Moosa Al-Khalaf (Supervisor) 